\title{
Evaluation of commercial probiotic lactic cultures against biofilm formation by Cronobacter sakazakii
}

\author{
Anubhav Jamwal ${ }^{1 *}$, Kavita Sharma ${ }^{1 *}$, Rajni Chauhan ${ }^{1}$, Saurabh Bansal ${ }^{1}$, Gunjan Goel ${ }^{1,2}$ \\ ${ }^{I}$ Department of Biotechnology and Bioinformatics, Jaypee University of Information Technology, Waknaghat; ${ }^{2}$ Department of Microbiology, \\ School of Interdisciplinary and Applied Life Sciences, Central University of Haryana, Mahendergarh, India
}

Background/Aims: Cronobacter sakazakii, an emergent pathogen is considered as a major concern to infants and neonates fed on reconstituted powdered infant milk formula. In conjunction with many other factors, biofilm forming capacity adds to its pathogenic potential. In view of the facts that infants are at highest risk to C. sakazakii infections, and emerging antibiotic resistance among pathogens, it is imperative to evaluate probiotic cultures for their efficacy against $C$. sakazakii. Therefore, pure probiotic strains were isolated from commercial probiotic products and tested for their antimicrobial and anti-biofilm activities against C. sakazakii. Methods: A total of 6 probiotic strains were tested for their antibiotic susceptibility followed by antimicrobial activity using cell-free supernatant (CFS) against C. sakazakii. The inhibitory activity of CFS against biofilm formation by $C$. sakazakii was determined using standard crystal violet assay and microscopic observations. Results: All the probiotic strains were sensitive to ampicillin, tetracycline, vancomycin and carbenicillin whereas most of the strains were resistant to erythromycin and novobiocin. Four of the 6 probiotic derived CFS possessed antimicrobial activity against C. sakazakii at a level of 40 $\mu \mathrm{L}$. A higher biofilm inhibitory activity $(>80 \%)$ was observed at initial stages of biofilm formation with weaker activity during longer incubation upto 48 hours (50\%-60\%). Conclusions: The study indicated the efficacy of isolated commercial probiotics strains as potential inhibitor of biofilm formation by C. sakazakii and could be further explored for novel bioactive molecules to limit the emerging infections of C. sakazakii. (Intest Res 2019;17:192-201)

Key Words: Probiotics; Cronobacter sakazakii; Biofilm; Antimicrobial

\section{INTRODUCTION}

Probiotics are live microorganisms when consumed in certain number provide health benefits beyond inherent basic nutrition. ${ }^{1}$ The most commonly used microbial genera in probiotic preparations are Lactobacillus, Bifidobacterium, Enterococcus, Bacillus, Streptococcus, and Saccharomyces. These strains have

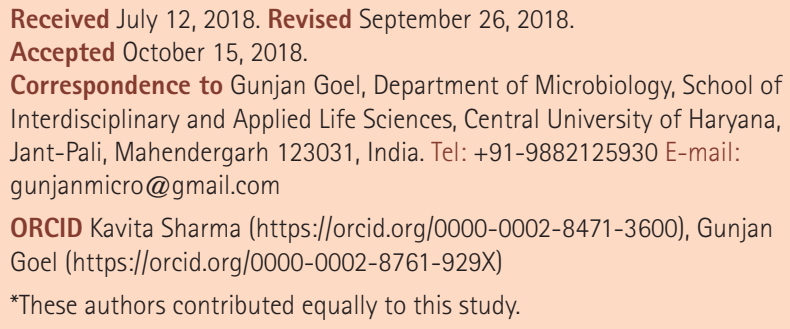

*These authors contributed equally to this study.

been reported for their effectiveness in varied clinical conditions-such as infantile diarrhea, necrotizing enterocolitis (NEC), antibiotic-associated diarrhea, relapsing Clostridium difficile colitis, Helicobacter pylori infections, inflammatory bowel and female urogenital infection. ${ }^{2}$ Apart from the GI disorders, the probiotics also promote a healthy gut microbiome, balanced immune function and enhanced nutrient absorption leading to a healthy host. The probiotics are also reported for their antimicrobial activities against the pathogens in GI tract via production of antimicrobial compounds (organic acids, hydrogen peroxide, bacteriocins), competing for nutrients and adhesion sites on host, decreasing luminal $\mathrm{pH}^{3}$

Cronobacter sakazakii is an emerging foodborne pathogen, causing life-threatening forms of neonatal meningitis, sepsis, and NEC in newborn and premature infants, ${ }^{4}$ with a fatality 
rate of $40 \%$ to $80 \%{ }^{5}$ In addition to its high-fatality rate, it may also result in severe neurological sequelae such as quadriplegia, hydrocephalus and retarded neural development in survivors. ${ }^{6}$ The environmental source of C. sakazakii is not evidently understood; however, the epidemiological studies implicate reconstituted powdered infant formula (PIF) as the possible source of transmission. ${ }^{7}$ Survival of bacteria in such a dry environment largely depends on the osmotic and/or dry stress resistance of the bacteria. ${ }^{8}$ Our laboratory has previously reported the prevalence of C. sakazakii among different food and environmental commodities. The isolates expressed different virulent factors such as hemolytic and chitinase activity along with the presence of genes encoding for hemolysin, outer membrane protein (ompA), Cronobacter plasminogen activator (сра) and ferric ion transporter protein (eitA). ${ }^{9-11}$ Apart from expression of these virulent traits, the isolates were able to form biofilm mediated by expression of acyl homoserine lactones as signaling molecules and genes encoding for cellulose synthesis. ${ }^{11}$

Although probiotic cultures have been reported for their antimicrobial activities against an array of pathogens leading the GI disorders, however, very few studies have been conducted to check the efficacy of probiotics against C. sakazakii. ${ }^{12,13}$ The commercial probiotic products are usually used to treat the diarrheal infections caused by gram negative pathogens, therefore, it is imperative to investigate the efficacy of these probiotics for their antimicrobial activities against C. sakazakii. To achieve this goal, the present study was designed to isolate pure strains of probiotics from commercial products and to investigate the anti-biofilm activities of cell-free supernatant (CFS) against biofilm forming C. sakazakii. The microscopic observations were also made to decipher the changes in the biofilm structure as induced by CFS.

\section{METHODS}

\section{Probiotic Products and Cultures}

A total of 6 different commercial probiotic supplements marketed in India were collected from local retailers (Table 1). All of the products except Yakult were in the powder formulation (sachets). The lactic acid bacteria from these products were isolated on de Man, Rogosa, Sharpe (MRS) agar plates. Colonies were differentiated according to their shape, size and other morphological features. The individual colonies obtained on MRS agar were further subcultured on MRS agar to get pure cultures. The cultures were tentatively identified based on Grams reaction and catalase test. The standard strain $C$. sakazakii ATCC 12868 used in the study was kindly gifted by Dr. Davis Tall Ben, FDA, USA. ${ }^{9}$ The indicator strain, available as glycerol stock was revived, subcultured in Tryptone Soya Broth (TSB) and checked for purity.

\section{Antibiotic Susceptibility Test for Isolated Probiotic Cultures}

The susceptibility of different isolates to antibiotics was determined by disk diffusion method as per the recommendations of National Committee for Clinical Laboratory Standards. ${ }^{14}$ The overnight grown cultures of probiotic isolates $\left(\mathrm{OD}_{600 \mathrm{~nm}}=0.5\right)$ were spread on Muller Hinton agar plates (HiMedia Laboratories, Mumbai, India). The antibiotic discs were placed on the cultured plates and incubated for 24 hours at $37^{\circ} \mathrm{C}$. The diameter of inhibition zones were measured and the results were expressed as sensitive (S) and resistant (R) as per EFSA, 2008. ${ }^{15}$

\section{Antimicrobial Activity of CFS against C. sakazakii}

The antimicrobial activity of CFS of overnight grown probiotic cultures was tested against C. sakazakii using agar well assay.

Table 1. Details of the Probiotic Products Procured for the Study

\begin{tabular}{|c|c|c|c|c|}
\hline Probiotic product & Compositions & $\begin{array}{l}\text { Date of } \\
\text { manufacture }\end{array}$ & Expire date & Lot no. \\
\hline Yakult & Lactobacillus casei strain Shirota & May 2017 & June 2017 & 11110MFA \\
\hline Vizylac & Lactobacillus sporogenes: 40 M spores & November 2017 & April 2019 & DVC-17096 \\
\hline Bifilac & $\begin{array}{l}\text { L. sporogene: } 50 \text { M cells, Streptococcus faecalis: } 30 \text { M cells, Clostridium } \\
\text { butyricum: } 2 \mathrm{M} \text { cells, Bacillus mesentericus: } 1 \mathrm{M} \text { cells }\end{array}$ & May 2017 & October 2018 & ALA711 \\
\hline Rinifol & Lactobacillus sp: 40 M spores & July 2017 & February 2019 & CDD110619 \\
\hline Vibact & $\begin{array}{l}\text { B. mesentericus: } 1 \mathrm{M} \text { spores, C. butyricum: } 2 \mathrm{M} \text { spores, L. sporogenes: } 5 \mathrm{M} \\
\text { spores, S. faecalis: } 30 \mathrm{M} \text { spores }\end{array}$ & October 2017 & March 2019 & BA702 \\
\hline Caplac & L. sporogenes: 60 M spores & November 2016 & April 2018 & 10916011000167 \\
\hline
\end{tabular}

M, millions; sp, species. 
The overnight grown probiotic strains in MRS broth were centrifuged $(2,000 \times g$ for 5 minutes) and the spent medium was collected in fresh tubes. The spent culture medium was filtered through $0.22 \mu \mathrm{M}$ nylon membrane filters (Millipore, Burlington, MA, USA). The CFS obtained was given 2 treatments: neutralization to a $\mathrm{pH}$ of 7.0 by addition of $1 \mathrm{~N}$ sodium hydroxide $(\mathrm{NaOH})$ and a heat treatment of $100^{\circ} \mathrm{C}$ for 5 minutes to obtain neutralized (N-CFS) and heat-treated (H-CFS) preparations respectively. All the $3 \mathrm{CFSs}$ (CFS, N-CFS, and H-CFS) were stored at $-80^{\circ} \mathrm{C}$ until further use. The un-inoculated MRS broth was taken as control.

For antimicrobial assay, the indicator strain of C. sakazakii was grown to exponential phase $\left(\mathrm{OD}_{600 \mathrm{~mm}}=0.5\right)$ in TSB and spread on the TSA plate. After spreading, wells were punched in the agar plates and the wells were filled with $100 \mu \mathrm{L}$ of all the 3 CFS preparations along with the control MRS broth. The plates were incubated at $35^{\circ} \mathrm{C}$ for 12 hours and the diameter of zones of inhibition ( $\mathrm{mm}$ ) was determined.

\section{Minimum Bactericical Concentration of CFS against C. sakazakii}

The minimum bactericidal concentrations (MBCs) of CFS were determined in microtitre plates by micro dilution assay as per the protocol of Clinical and Laboratory Standards Institute. The untreated CFS was diluted in the range of 0.13 to 0.250 of total volume in TSB. Briefly, a bacterial inoculum (10 $\mu \mathrm{L}, \mathrm{OD}_{600 \mathrm{~nm}}=0.5$ ) was added the microtitre plates containing TSB, followed by addition of different concentrations of CFS. TSB alone in the wells was used as a negative control. The final volume of each well was $250 \mu \mathrm{L}$. The plates were incubated at $37^{\circ} \mathrm{C}$ for 24 hours and absorbance was measured at 600 $\mathrm{nm}$. Among the tested probiotic derived CFS, only 4 CFS samples indicated effective inhibition of growth of C. sakazakii at a concentration of $50 \mu \mathrm{L}$, therefore, for further biofilm inhibition assay, only 4 strains were tested.

\section{Anti-Biofilm Activity of the CFS}

The anti-biofilm potential of CFS from isolated probiotic strains was determined in polystyrene flat-bottom 96-well microtiter plates using crystal violet (CV) staining assay. ${ }^{16}$ In brief, a 10 $\mu \mathrm{L}$ cell suspension of $C$. sakazakii indicator strain $\left(\mathrm{OD}_{600 \mathrm{~mm}}=0.5\right)$ was incubated along with CFS ( $40 \mu \mathrm{L}$ each) for inhibition of biofilm formation at different time period. The plates were incubated for 48 hours at $37^{\circ} \mathrm{C}$. After a time, interval of 12, 24, and 48 hours of incubation, the planktonic cell suspension was aspirated and wells were washed with phosphate buff- ered saline $(1 \times$ PBS $)$ to remove loosely adherent cells. The surface adhered cells were stained with $200 \mu \mathrm{L}$ of $0.2 \%$ CV solution for 15 minutes at room temperature. Following staining, CV solution was decanted and the stained cells were solubilized with $250 \mu \mathrm{L}$ of $33 \%$ glacial acetic acid. The biofilm biomass was then quantified by measuring the intensity of $\mathrm{CV}$ at $\mathrm{OD}_{570 \mathrm{~nm}}$ using a Multiskan Go microplate reader (Thermo Fischer Scientific, Waltham, MA, USA).

The anti-biofilm activity (\%) was calculated following the formula: anti-biofilm activity $(\%)=\left(\right.$ control $\mathrm{OD}_{570 \mathrm{~nm}}-$ test $\mathrm{OD}_{570 \mathrm{~nm}} /$ control $\left.\mathrm{OD}_{570 \mathrm{~nm}}\right) \times 100$. The un-inoculated MRS broth was taken as negative control.

\section{Visualization of Biofilms}

\section{1) Light Microscopic Analysis}

The biofilm of indicator C. sakazakii strain was developed on glass cover slips $(1 \times 1 \mathrm{~cm})$. The strain was grown in 6 -well tissue culture plate containing sterile glass cover slips, TSB (1,680 $\mu \mathrm{L} /$ well) and CFSs from different lactic acid bacterial strains ( $240 \mu \mathrm{L} /$ well). The wells without CFS were taken as control. The plates were incubated for 48 hours at $37^{\circ} \mathrm{C}$. After 24 hours, the planktonic cells were removed and cover slips were rinsed with phosphate buffer (pH 6.5) and were stained with $0.2 \%$ CV solution for 30 minutes. The excess of stain was washed twice with distilled water and the slides were air dried. The stained cover slips were visualized under light microscope at ×40 magnification (Olympus Microscope, Tokyo, Japan).

\section{2) Fluorescent Microscopy}

For the fluorescent microscopic images, the biofilms were treated as discussed before. After the treatment of biofilms, the cover slips were stained with LIVE/DEAD Bac Light bacterial viability kit (L10316; Invitrogen-Molecular Probes, Waltham, MA, USA). The kit included mixture of 2 fluorescence dyes: SYTO 9 (green-fluorescent nucleic acid stain) and propidium iodide (red-fluorescent nucleic acid stain). The viable bacterial cells with a healthy or intact membrane stain green, whereas dead cells or cells with a damaged membrane stain fluorescent red. The stained coverslips were observed at $\times 400$ magnification using a fluorescence microscope BX53 (Olympus Microscopy) equipped with imaging system Qiclick ${ }^{\mathrm{TM}}$ (Olympus).

\section{Statistical Analysis}

Each experiment was conducted in triplicate at 3 different time points and the data were represented as mean values $(n=9)$. The statistical significance among the treatments was 
conducted using ANOVA and Tukey multiple comparison test $(P<0.05)$ with SPSS version 21.0 (IBM Corp., Armonk, NY, USA).

\section{RESULTS}

\section{Probiotic Bacterial Isolates}

A total of 6 distinct colonies were obtained on MRS agar plates. All the cultures were Gram positive rods, responded negative to catalase test except one isolate from Bifilac which was Gram positive cocci. The isolates tested in the present study were Streptococcus faecalis (Bifilac), Lactobacillus sporogenes (Vizylac, Vibact, Caplac), Lactobacillus sp (Rinifol) and Lactobacillus casei strain Shirota (Yakult) (Table 1).

\section{Antibiotic Susceptibility of Lactic Cultures}

The isolated cultures were tested for their antibiotic susceptibility pattern. Varying pattern of antibiotic susceptibility was obtained with probiotic culture isolated from commercial probiotic products. The antibiotic susceptibility profile is pre- sented in Table 2. Most of the strains were resistant to erythromycin and novobiocin. All the isolated strains were sensitive to ampicillin, tetracycline, vancomycin and carbenicillin.

\section{Antimicrobial Activity of CFS against C. sakazakii}

The antimicrobial activity of probiotic isolates was determined by determining the growth inhibition of $C$. sakazakii in the presence of CFS of probiotic isolates (Table 3). The results indicated that untreated and heat treated CFS from all the cultures inhibited the growth of C. sakazakii with an inhibition zone ranging between 10 and $14 \mathrm{~mm}$ (Supplementary Fig. 1). No zone of inhibition was observed using neutralized CFS expect for the L. sporogenes obtained from Vizylac.

\section{MBC of CFS against C. sakazakii}

The MBC assay revealed different susceptibilities of indicator organisms to the CFS of probiotic strains, and exhibited potent inhibitory effect as represented as MBC values (Table 4). From this MBC assay, it was observed that a $50 \mu \mathrm{L}$ concentra-

Table 2. Antibiotic Susceptibility of Probiotic Cultures Using Agar Disk Diffusion Assay

\begin{tabular}{lcccccccccccccccc}
\hline Isolates & Source & C & RIF & AMC & B & TE & HLG & E & NV & CB & VA & CEP & AK & CD & S & AMP \\
\hline Lactobacillus caseistrain Shirota & Yakult & S & S & R & S & S & S & S & R & S & S & S & S & R & S & S \\
Lactobacillus sporogenes & Vizylac & S & R & S & R & S & R & S & R & S & S & R & I & R & I & S \\
Streptococcus faecalis & Bifilac & S & S & S & R & S & S & R & R & S & S & I & I & R & R & S \\
Lactobacillusspecies & Rinifol & R & R & R & R & S & S & R & R & S & S & S & I & R & I & S \\
L. sporogenes & Vibact & S & S & S & S & S & S & R & I & S & S & S & I & R & I & S \\
L. sporogenes & Caplac & S & S & S & R & S & S & R & R & S & S & S & I & R & I & S \\
\hline
\end{tabular}

In the table, $\mathrm{S}$ is susceptible, $\mathrm{R}$ is resistant, and I is intermediate.

C, chloramphenicol 30 units/disk; RIF, rifampicin $5 \mu \mathrm{g} /$ disk; AMC, amoxiclav $30 \mu \mathrm{g} /$ disk; B, bacitracin 10 units/disk; TE, tetracycline, $30 \mu \mathrm{g} /$ disk; HLG,

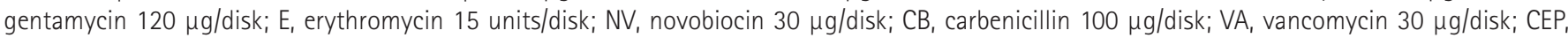
cephalothin $30 \mu \mathrm{g} /$ disk; $\mathrm{AK}$, amikacin $30 \mu \mathrm{g} /$ disk; CD, clindamycin $2 \mu \mathrm{g} /$ disk; S, streptomycin $10 \mu \mathrm{g} / \mathrm{disk}$; AMP, ampicillin $30 \mu \mathrm{g} /$ disk.

Table 3. Antimicrobial Activities in Terms of Zone of Inhibition $(\mathrm{mm})$ of Probiotic Isolates against Cronobacter sakazakii Using Agar Well Assay

\begin{tabular}{llccc}
\hline Isolates & Source & Supernatant & Heat neutralized & pH neutralized \\
\hline Lactobacillus species & Yakult & $12.3^{\mathrm{a}, \mathrm{x}} \pm 0.5$ & $11.3^{\mathrm{a}, \mathrm{x}} \pm 1.1$ & - \\
Lactobacillus sporogenes & Vizylac & $12.6^{\mathrm{a,x}} \pm 1.1$ & $11.3^{\mathrm{a}, \mathrm{x}} \pm 1.1$ & $9.6^{\mathrm{b}, \mathrm{y}} \pm 0.5$ \\
Streptococcus faecalis & Bifilac & $12.3^{\mathrm{a}, \mathrm{x}} \pm 0.5$ & $11.3^{\mathrm{a}, \mathrm{x}} \pm 0.5$ & - \\
Lactobacillus species & Rinifol & $10.0^{\mathrm{b}, \mathrm{x}} \pm 0.1$ & $10.3^{\mathrm{b}, \mathrm{x}} \pm 0.5$ & - \\
L. sporogenes & Vibact & $10.6^{\mathrm{b}, \mathrm{x}} \pm 0.5$ & $10.0^{\mathrm{b}, \mathrm{x}} \pm 0.1$ & - \\
L. sporogenes & Caplac & $14.3^{\mathrm{c}, \mathrm{x}} \pm 0.5$ & $12.3^{\mathrm{a}, \mathrm{y}} \pm 0.5^{-}$ & - \\
\hline
\end{tabular}

${ }^{a-c}$ Means in the column with same superscript letter are not significantly different as measured by 2-sided Tukey post-hoc range test between replications.

${ }^{x, y}$ Means in the row with same superscript letter are not significantly different as measured by 2-sided Tukey post-hoc range test between replications. 
Table 4. Minimum Bactericidal Concentration Based on Optical Density $\left(\mathrm{OD}_{600 \mathrm{~nm}}\right)$ of CFS of Probiotic Isolates on Growth of Cronobacter sakazakii

\begin{tabular}{|c|c|c|c|c|}
\hline Isolates & Source & CFS $(30 \mu \mathrm{L})$ & CFS $(40 \mu \mathrm{L})$ & CFS $(50 \mu \mathrm{L})$ \\
\hline Lactobacillus species & Yakult & $3.05^{a, x} \pm 0.08$ & $2.20^{b, y} \pm 0.15$ & $0.26^{b, z} \pm 0.12$ \\
\hline Lactobacillus sporogenes & Vizylac & $2.99^{a, x} \pm 0.10$ & $3.01^{a, x} \pm 0.10$ & $3.01^{a, x} \pm 0.10$ \\
\hline Streptococcus faecalis & Bifilac & $2.53^{a, x} \pm 0.35$ & $1.84^{c, y} \pm 0.25$ & $0.43^{b, z} \pm 0.05$ \\
\hline Lactobacillus species & Rinifol & $2.89^{a, x} \pm 0.20$ & $2.91^{\mathrm{a}, \mathrm{x}} \pm 0.15$ & $2.81^{\mathrm{a}, \mathrm{x}} \pm 0.21$ \\
\hline L.sporogenes & Vibact & $2.43^{\mathrm{a}, \mathrm{x}} \pm 0.30$ & $1.61^{c, y} \pm 0.20$ & $0.02^{b, z} \pm 0.04$ \\
\hline L. sporogenes & Caplac & $2.59^{a, x} \pm 0.34$ & $1.70^{c, y} \pm 0.14$ & $0.21^{b, z} \pm 0.31$ \\
\hline
\end{tabular}

${ }^{a-c}$ Means in the column with same superscript letter are not significantly different as measured by 2-sided Tukey post-hoc range test between replications.

${ }^{x, 2}$ Means in the row with same superscript letter are not significantly different as measured by 2-sided Tukey post-hoc range test between replications. CFS, cell-free supernatant.

Table 5. Biofilm Inhibition Potential (\%) of Cell-Free Supernatant from Probiotic Isolates at Different Stages of Biofilm Formation by Cronobacter sakazakii

\begin{tabular}{|c|c|c|c|c|}
\hline Isolates & Source & 12 Hours & 24 Hours & 48 Hours \\
\hline Lactobacillus species & Yakult & $82^{a, x} \pm 2.6$ & $57^{b, y} \pm 3.3$ & $56^{b, y} \pm 2.8$ \\
\hline Streptococcus faecalis & Bifilac & $85^{a, x} \pm 1.2$ & $62^{b, y} \pm 5.1$ & $62^{\text {b.y }} \pm 5.7$ \\
\hline L. sporogenes & Caplac & $84^{a, x} \pm 3.1$ & $60^{b, y} \pm 4.2$ & $57^{b, y} \pm 4.1$ \\
\hline
\end{tabular}

${ }^{a-c}$ Means in the column with same superscript letter are not significantly different as measured by 2-sided Tukey post-hoc range test between replications.

${ }^{x, y}$ Means in the row for each time interval with same superscript letter are not significantly different as measured by 2-sided Tukey post-hoc range test between replications.

tion of CFS resulted in complete inhibition of C. sakazakii, therefore, a concentration of $40 \mu \mathrm{L}$ of CFS was used for further experiments.

\section{Biofilm Inhibition Potential}

The biofilm inhibitory activities of the CFS of lactic cultures at diferent time interval is presented in Table 5. Similar to the antimicrobial activity, the CFS possessed varying degree of biofilm inhibitory activity. The higher inhibiton was observed at intial incubation of 12 hours (>80\%) after which there was weaker anti-biofilm activity after 24 and 48 hours of incubation (50\%-60\%) for all the strains $(P>0.05)$.

\section{Microscopic Observations}

The stained microscopic observations revealed a clear difference between the treated and the control biofilm of C. sakazakii (Figs. 1 and 2). The microscopic images showed that the CFS reduced the adhesion of bacterial cell to the glass cover slips. The control slides indicated a uniform, consistent and well-developed biofilm whereas the slides treated with CFS were observed to have scattered biofilm.

\section{DISCUSSION}

C. sakazakii is an emerging life-threatening food borne pathogen usually implicated in reconstituted PIF and dairy products resulting in life-threatening forms of neonatal meningitis, sepsis, and NEC in newborn and premature infants and immune-compromised adults. ${ }^{9}$ The pathogen is also able to survive under harsh food processing conditions due its ability to form biofilm on abiotic surfaces. ${ }^{9,16-19}$ As the pathogen is mainly implicated in the milk formula therefore, the lactic acid bacteria usually present in dairy sources can be used to prevent the growth and biofilm inhibitory activities. Therefore, this study was designed to test the probiotic cultures derived from commercial preparations used in diarrheal infections, for their antimicrobial activities against C. sakazakii.

All the isolates obtained from commercial preparation belonged to lactic acid bacteria group. Regarding the safer use of probiotics for human consumption, horizontal gene transfer 


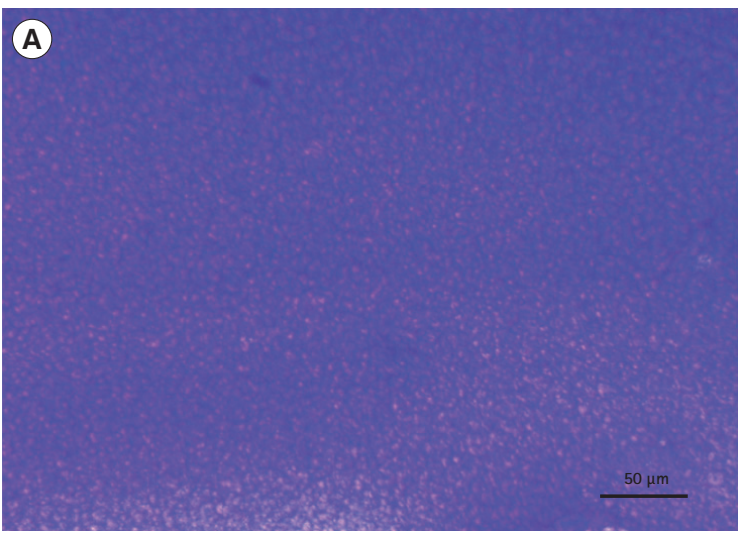

Control

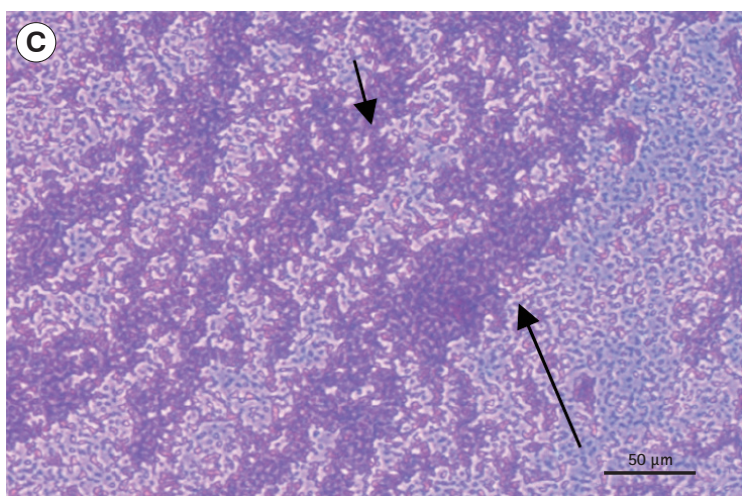

$24 \mathrm{hr}$

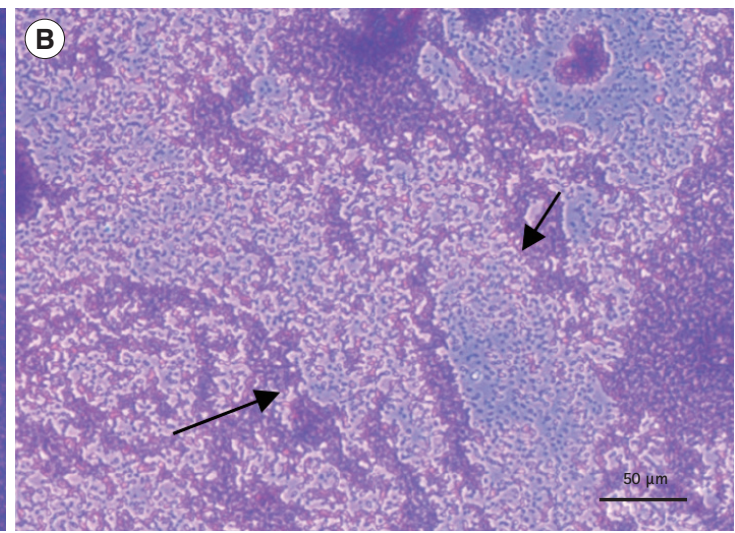

$12 \mathrm{hr}$

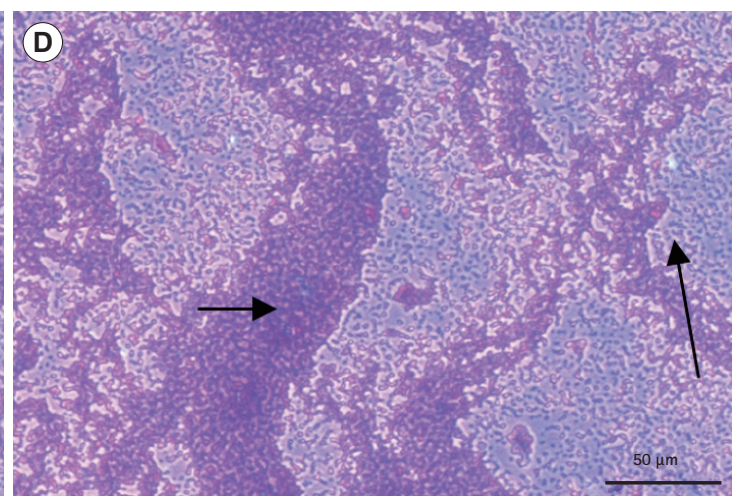

$48 \mathrm{hr}$

Fig. 1. (A-D) Effect of cell-free supernatant from probiotic cultures on biofilm formation by Cronobacter sakazakii stained with crystal violet staining (arrows indicate disrupted biofilm at different hours of incubation) at $\times 40$.

of antibiotic resistance gene harbored by these cultures is of major concern, therefore, the determination of antibiotic susceptibility is one of the important safety concerns for the probiotic cultures applications. ${ }^{20}$ All the lactic cultures obtained from commercial preparation were tested for their susceptibility pattern to different antibiotic. All the probiotic strains were sensitive to ampicillin, tetracycline, vancomycin and carbenicillin whereas most of the strains were resistant to erythromycin and novobiocin. The Lactobacillus isolates from Yakult and Vibact were the most sensitive strains to the tested antibiotics. The isolate obtained from Rinifol was the strain possessing resistance towards many tested antibiotics. However, as reported for the lactic cultures towards their intrinsic resistance to vancomycin, we did not observe any resistance to vancomycin in isolates. The intrinsic resistance in the probiotic strains may be considered as beneficial as it will allow them to maintain the microbial ecological balance in gut during antibiotic therapy. ${ }^{21}$

The spent culture medium from isolated probiotics was further tested for its antimicrobial and anti-biofilm activities against the $C$. sakazakii as indicator strain. Along with the untreated CFS, all the heat neutralized cell supernatants of all cultures also inhibited the growth of the indicator strain. The results indicated that the inhibitory substance(s) in the CFSs were heatresistant as activity was not abolished after heat treatment. Except neutralized CFS from $L$. sporogenes isolated from Vizylac, no zone of inhibition was observed with other cultures indicating that there might be a possible role of metabolites/products such as bacteriocins for this culture other than organic acids which resulted in antimicrobial activities. Results indicated that in most of the cases the inhibitory effect of lactobacilli may be resulted from the production of antibacterial organic acid molecules or antibacterial agents such as bacteriocin that are active in acid condition. ${ }^{22}$ Many other studies have reported that the inhibition of food-borne pathogens by probiotic lactic cultures can be mediated by competitive exclusion or production of antimicrobial metabolites such as organic acids, hydrogen peroxide and antimicrobial peptides (e.g., bacteriocins) ${ }^{23,24}$ Regarding the antimicrobial activities of lactic cultures against $C$. sakazakii; Hayes et al., ${ }^{25}$ assessed the activi- 
A
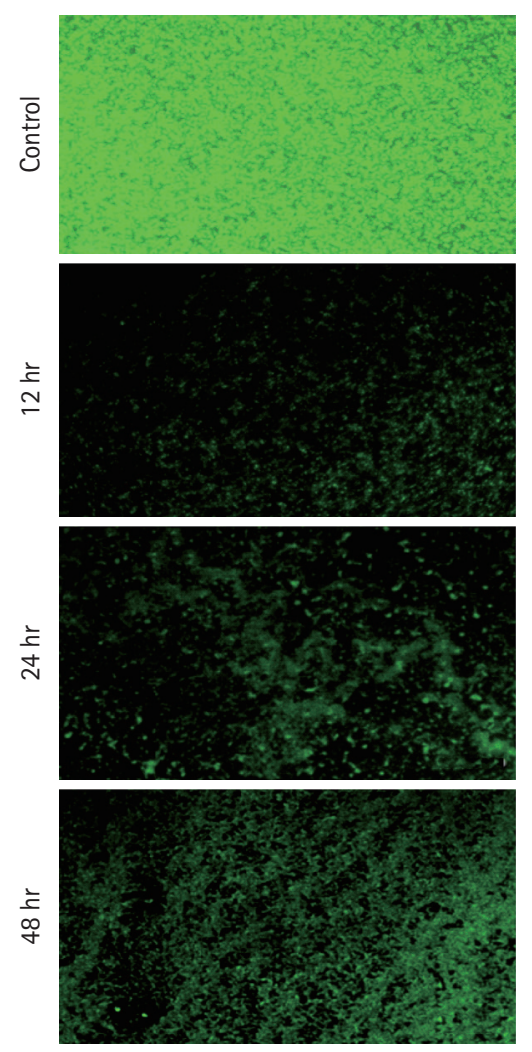

B
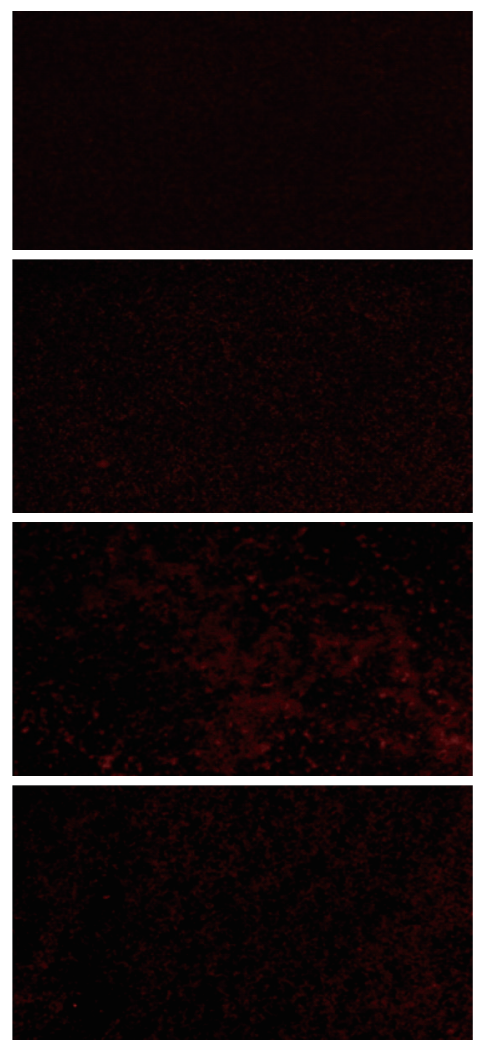

C

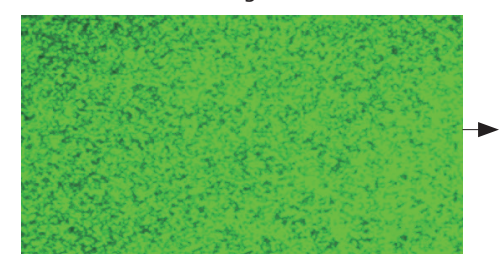

Strong biofilm
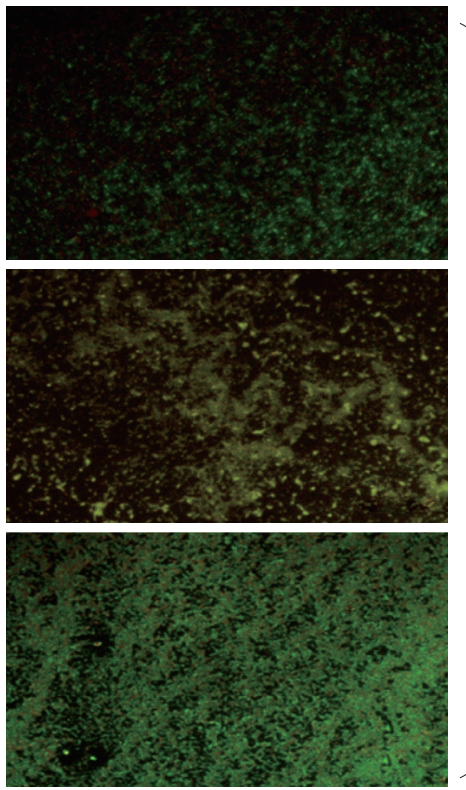

Disrupted biofilm

Fig. 2. Fluorescent microscopic images of effect of cell-free supernatant (CFS) from probiotics on biofilms of Cronobacter sakazakii grown at different stages using live-dead stain at $50 \mu \mathrm{m}$. (A) Stained with SYTO-9, (B) slides stained with propidium lodide (PI), (C) merged image.

ty of sodium caseinate fermentate (obtained from the fermentation of sodium caseinate with the proteolytic strain of Lactobacillus acidophilus DPC 6026) for decreasing the numbers of Cronobacter spp. in reconstituted PIF. At higher final concentrations of fermentate $(3.33 \% \mathrm{w} / \mathrm{v})$, numbers were completely eliminated from $6 \operatorname{logs} \mathrm{CFU} / \mathrm{mL}$ over an incubation time of 60 minutes. Similarly, Awaisheh et al., ${ }^{26}$ reported the antimicrobial activity of pH-neutralized CFS of L. acidophilus or L. casei (isolated from faces of infants) against tested C. sakazakii. They also reported that the inhibition was due to the production of bacteriocins by L. acidophilus and L. casei. Recently, Kim et al., ${ }^{27}$ has also reported the antimicrobial activity of Lactobacillus kefiri DH5 against C. sakazakii due to production of organic acids. The CFS was reported to disrupt the cellular membrane integrity and induce leakage of cytoplasmic materials including nucleic acid and proteins. Yi et al., ${ }^{28}$ have reported the antagonistic activity of a bacteriocin preparation from $\mathrm{Lac}$ tobacillus crustorum MN047 disrupting the integrity of cell envelope leading to cell membrane permeabilization.

As reported before, the C. sakazakii strain used in the pres- ent study start forming biofilm after 12 hours of inucbation and a mature biofilm is formed after 48 hours of incubation, ${ }^{16}$ therefore, the anti-biofilm activity of the probiotic isolates against C. sakazakii was evaluated at different stages of biofilm formation (12-48 hours). The basis for the suppression of biofilm formation by C. sakazakii with CFS from different strains may be attributed to the presence of certain metabolites in the CFS as previously bacteriocins/metabolic products from lactobacilli are reported to induce unfavourable situation for the development of biofilm by pathogenic bacteria. ${ }^{29-32}$ The CFS tested in this study possessed higher inhibition at initial stages of biofilm formation and were weaker to suppress/eliminate the biofilm at later stages, suggested the effects on the biofilms might be physicochemical or more particularly, interfacial in nature. The biosurfactants in metabolites liberated by lactobacilli are reported to interfere with the adhesion of cells, since these decrease the hydrophobicity of the surface substratum and obstruct with progressions related to microbial adhesion and desorption. ${ }^{33-35}$ The ability to disrupt biofilm formation process of C. sakazakii with CFS from lactic cultures 
was also assessed by microscopic observations. Representative light microscopic and fluorescent images indicated disruptive effects of CFS by interfering with bacterial attachment and destabilizing the biofilm matrix. Candela et al. ${ }^{36}$ reported that different strains of probiotic bacteria (Lactobacillus plantarum Bar10, L. acidophilus Bar13, Bifidobacterium lactis Bar30 and Bifidobacterium longum Bar33) were capable of obstructing the adhesion sites for enteropathogens Escherichia coli H10407 and Salmonella typhimurium on Caco-2 cell line. In another study, the extracellular polysaccharides liberated from L. acidophilus A4 declined biofilms of enterohemorrhagic $E$. coli in vitro by affecting genes related to chemotaxis and curli production. ${ }^{37}$ These may also alter the biofilm integrity via interference with cell-to-cell aggregation and surface attachment processes. This effect may be mediated by the exopolysaccharides release by lactic cultures or by the physicochemical properties of their cell surface. ${ }^{38,39}$

In conclusions, the pathogens such as C. sakazakii possessing biofilm mediated tolerance to different food processing conditions continue to pose risk to human health. For the effective control of biofilm formation process of C. sakazakii, probiotic lactobacilli are suitable candidate as described in this study. The outcome of this study will provide appropriate measures to be taken by health care providers for recommending probiotics against infection by the pathogen. Such information will be of interest to regulatory agencies for food production units where heating processes or other processing conditions are not sufficient to kill the target pathogen. However, further studies are needed to identify the functional moiety in the crude CFS to promote its application in biocontrol of biofilm mediated stress responses in C. sakazakii.

\section{FINANCIAL SUPPORT}

The authors received no financial support for the research, authorship, and/or publication of this article.

\section{CONFLICT OF INTEREST}

No potential conflict of interest relevant to this article was reported.

\section{AUTHOR CONTRIBUTION}

Jamwal A, Sharma K, Goel G planned, performed and drafted the manuscript. Chauhan R, Bansal S, Goel G reviewed and edited the manuscript. All the authors approved the final manuscript.

\section{REFERENCES}

1. Kumar N, Raghavendra M, Tokas J, Singal HR. Flavor addition in dairy products: health benefits and risks. In: Watson RR, Cllier RJ, Preedy VR, eds. Nutrients in dairy and their implications on health and disease. Cambridge: Academic Press, 2018; 123-135.

2. Rondanelli M, Faliva MA, Perna S, Giacosa A, Peroni G, Castellazzi AM. Using probiotics in clinical practice: where are we now? A review of existing meta-analyses. Gut Microbes 2017; 8:521-543.

3. Sánchez B, Delgado S, Blanco-Míguez A, Lourenço A, Gueimonde M, Margolles A. Probiotics, gut microbiota, and their influence on host health and disease. Mol Nutr Food Res 2017; 61: 201600240 .

4. Beuchat LR, Kim H, Gurtler JB, Lin LC, Ryu JH, Richards GM. Cronobacter sakazakii in foods and factors affecting its survival, growth, and inactivation. Int J Food Microbiol 2009;136: 204-213

5. Bowen AB, Braden CR. Invasive Enterobacter sakazakii disease in infants. Emerg Infect Dis 2006;12:1185-1189.

6. Forsythe SJ. Enterobacter sakazakii and other bacteria in powdered infant milk formula. Matern Child Nutr 2005;1:44-50.

7. Umeda NS, de Filippis I, Forsythe SJ, Brandão ML. Phenotypic characterization of Cronobacter spp. strains isolated from foods and clinical specimens in Brazil. Food Res Int 2017;102:61-67.

8. Osaili T, Forsythe S. Desiccation resistance and persistence of Cronobacter species in infant formula. Int J Food Microbiol 2009;136:214-220.

9. Singh N, Goel G, Raghav M. Insights into virulence factors determining the pathogenicity of Cronobacter sakazakii. Virulence 2015;6:433-440.

10. Singh N, Patil A, Prabhune AA, Raghav M, Goel G. Diverse profiles of N-acyl-homoserine lactones in biofilm forming strains of Cronobacter sakazakii. Virulence 2017;8:275-281.

11. Singh N, Raghav M, Narula S, Tandon S, Goel G. Profiling of virulence determinants in Cronobacter sakazakii isolates from different plant and environmental commodities. Curr Microbiol 2017;74:560-565.

12. Collado MC, Isolauri E, Salminen S. Specific probiotic strains and their combinations counteract adhesion of Enterobacter sakazakii to intestinal mucus. FEMS Microbiol Lett 2008;285: 58-64. 
13. Sharma G, Prakash A. Susceptibility of Cronobacter sakazakii to plant products, antibiotics, and to lactic acid bacteria. Int J Nutr Pharmacol Neurol Dis 2013;3:263-268.

14. Clinical and Laboratory Standards Institute. Performance standards for antimicrobial susceptibility testing. 27th ed. CLSI supplement M100. Wayne: CLSI, 2017.

15. European Food Safety Authority (EFSA). Technical guidance: update of the criteria used in the assessment of bacterial resistance to antibiotics of human or veterinary importance. EFSA J 2008;6:732.

16. Singh N, Patil A, Prabhune A, Goel G. Inhibition of quorumsensing-mediated biofilm formation in Cronobacter sakazakii strains. Microbiology 2016;162:1708-1714.

17. Iversen C, Forsythe S. Isolation of Enterobacter sakazakii and other Enterobacteriaceae from powdered infant formula milk and related products. Food Microbiol 2004;21:771-777.

18. Lehner A, Riedel K, Eberl L, Breeuwer P, Diep B, Stephan R. Biofilm formation, extracellular polysaccharide production, and cell-to-cell signaling in various Enterobacter sakazakii strains: aspects promoting environmental persistence. J Food Prot 2005;68:2287-2294.

19. Kim H, Ryu JH, Beuchat LR. Attachment of and biofilm formation by Enterobacter sakazakii on stainless steel and enteral feeding tubes. Appl Environ Microbiol 2006;72:5846-5856.

20. Georgieva R, Yocheva L, Tserovska L, et al. Antimicrobial activity and antibiotic susceptibility of Lactobacillus and Bifidobacterium spp. intended for use as starter and probiotic cultures. Biotechnol Biotechnol Equip 2015;29:84-91.

21. Neut C, Mahieux S, Dubreuil LJ. Antibiotic susceptibility of probiotic strains: is it reasonable to combine probiotics with antibiotics? Med Mal Infect 2017;47:477-483.

22. Shokri D, Khorasgani MR, Mohkam M, Fatemi SM, Ghasemi Y, Taheri-Kafrani A. The inhibition effect of Lactobacilli against growth and biofilm formation of Pseudomonas aeruginosa. Probiotics Antimicrob Proteins 2018;10:34-42.

23. Kivanc M, Yilmaz M, Cakir E. Isolation and identification of lactic acid bacteria from boza, and their microbial activity against several reporter strains. Turk J Biol 2011;35:313-324.

24. Denkova RS, Yanakieva VB, Denkova ZR, Hristozova P. Inhibitory activity of the probiotic strain Bifidobacterium bifidum Bif. 4 of human origin against pathogens. J Food Packag Sci Tech Technol 2013:(2):21-25.

25. Hayes M, Barrett E, Ross RP, Fitzgerald GF, Hill C, Stanton C. Evaluation of an antimicrobial ingredient prepared from a Lactobacillus acidophilus casein fermentate against Enterobacter sakazakii. J Food Prot 2009;72:340-346.
26. Awaisheh SS, Al-Nabulsi AA, Osaili TM, Ibrahim S, Holley R. Inhibition of Cronobacter sakazakii by heat labile bacteriocins produced by probiotic LAB isolated from healthy infants. J Food Sci 2013;78:M1416-M1420.

27. Kim DH, Jeong D, Song KY, Kang IB, Kim H, Seo KH. Culture supernatant produced by Lactobacillus kefiri from kefir inhibits the growth of Cronobacter sakazakii. J Dairy Res 2018; 85:98-103.

28. Yi L, Dang Y, Wu J, et al. Purification and characterization of a novel bacteriocin produced by Lactobacillus crustorum MN047 isolated from koumiss from Xinjiang, China. J Dairy Sci 2016; 99:7002-7015.

29. Drenkard E, Ausubel FM. Pseudomonas biofilm formation and antibiotic resistance are linked to phenotypic variation. Nature 2002;416:740-743.

30. François ZN, Marie KP, Noëlle TA, Emeric GW. Antimicrobial activity of a bacteriocin produced by Lactobacillus plantarum $29 \mathrm{~V}$ and strain's viability in palm kernel oil. Int J Nutr Food Sci 2013;2:102-108.

31. Rao KP, Chennappa G, Suraj U, Nagaraja H, Raj AP, Sreenivasa MY. Probiotic potential of lactobacillus strains isolated from sorghum-based traditional fermented food. Probiotics Antimicrob Proteins 2015;7:146-156.

32. Schaffer AC, Lee JC. Vaccination and passive immunisation against Staphylococcus aureus. Int J Antimicrob Agents 2008; 32 Suppl 1:S71-S78.

33. Saravanakumari P, Mani K. Structural characterization of a novel xylolipid biosurfactant from Lactococcus lactis and analysis of antibacterial activity against multi-drug resistant pathogens. Bioresour Technol 2010;101:8851-8854

34. Shokouhfard M, Kermanshahi RK, Shahandashti RV, Feizabadi MM, Teimourian S. The inhibitory effect of a Lactobacillus acidophilus derived biosurfactant on biofilm producer Serratia marcescens. Iran J Basic Med Sci 2015;18:1001-1007.

35. Sambanthamoorthy K, Feng X, Patel R, Patel S, Paranavitana C. Antimicrobial and antibiofilm potential of biosurfactants isolated from lactobacilli against multi-drug-resistant pathogens. BMC Microbiol 2014;14:197.

36. Candela M, Perna F, Carnevali P, et al. Interaction of probiotic Lactobacillus and Bifidobacterium strains with human intestinal epithelial cells: adhesion properties, competition against enteropathogens and modulation of IL-8 production. Int J Food Microbiol 2008;125:286-292.

37. Kim Y, Oh S, Kim SH. Released exopolysaccharide (r-EPS) produced from probiotic bacteria reduce biofilm formation of enterohemorrhagic Escherichia coli O157:H7. Biochem Bio- 
phys Res Commun 2009;379:324-329.

38. Bernal P, Llamas MA. Promising biotechnological applications of antibiofilm exopolysaccharides. Microb Biotechnol 2012;5:670-673.
39. Patel S, Majumder A, Goyal A. Potentials of exopolysaccharides from lactic acid bacteria. Indian J Microbiol 2012;52:312. 
See "Evaluation of commercial probiotic lactic cultures against biofilm formation by Cronobacter sakazakii" on page 192-201.

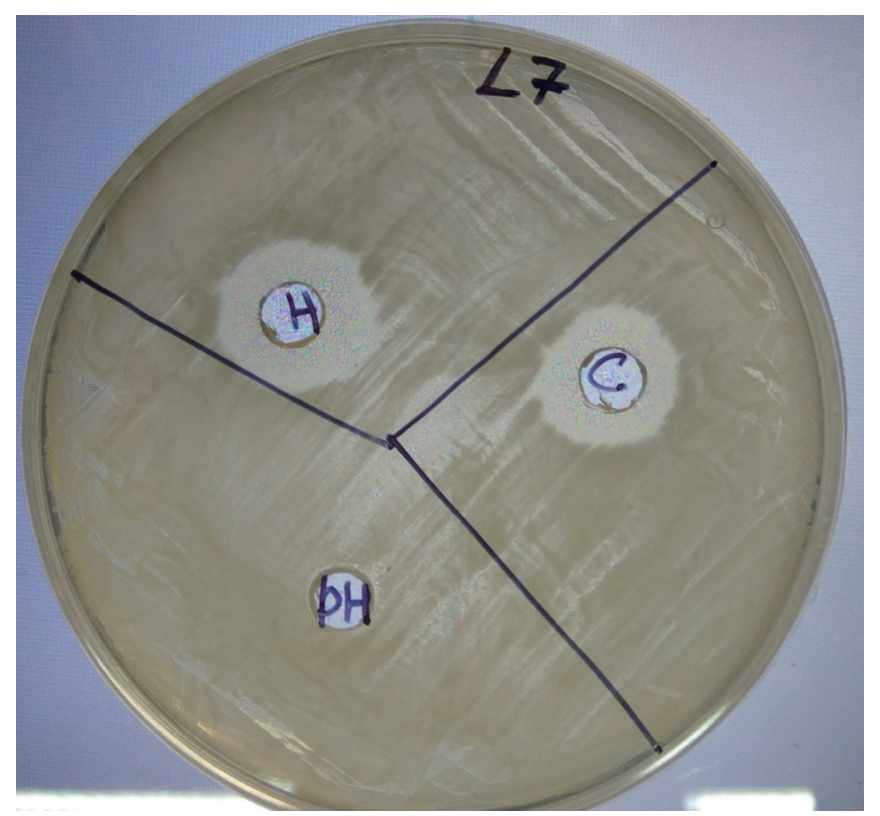

Supplementary Fig. 1. Antimicrobial activity of different fractions of cell-free supernatant (CFS) against Cronobacter sakazakii

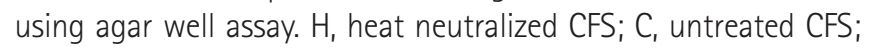
$\mathrm{pH}, \mathrm{pH}$ neutralized CFS. 